\title{
PEAK SETS FOR LIPSCHITZ FUNCTIONS
}

\author{
W. P. NOVINGER AND D. M. OBERLIN ${ }^{1}$
}

ABSTRACT. We study the peak sets for the algebras of functions analytic in the unit disc $D$ and satisfying a Lipschitz condition on $\partial D$.

Let $D$ denote the open unit disc in the complex plane, let $\bar{D}$ denote its closure, and let $\partial D$ denote its boundary. For $0<\alpha \leqslant 1$, let Lip $\alpha$ be the algebra of complex-valued functions $f$ analytic on $D$, continuous on $\bar{D}$, and satisfying a Lipschitz condition of order $\alpha$ on $\partial D$ :

$$
|f(z)-f(w)| \leqslant K|z-w|^{\alpha} \quad(z, w \in \partial D) .
$$

Say that a function $f$ defined on $\bar{D}$ peaks on $E \subseteq \partial D$ if $f=1$ on $E$ and $|f|<1$ on $\bar{D} \sim E$. Finally, say that $E \subseteq \partial D$ is a peak set for Lip $\alpha$ if some $f \in \operatorname{Lip} \alpha$ peaks on $E$. We are interested in characterizing the peak sets for Lip $\alpha$. For $\alpha=1$, the situation is easily described. It follows from our Theorem 1 that a peak set for Lip 1 must be finite. On the other hand, a result of B. A. Taylor and D. L. Williams [7] shows that any finite subset of $\partial D$ is a peak set for Lip 1. (In fact, [7] shows that the peaking function may be chosen to be infinitely differentiable on $\partial D$.) For $0<\alpha<1$, though, the situation seems more difficult, and we do not have such a characterization. Theorems 2 and 3 below give, respectively, sufficient and necessary conditions that $E \subseteq \partial D$ be a peak set for Lip $\alpha(0<\alpha<1)$. These conditions lend support to our conjecture of a necessary and sufficient condition, which we give at the end of this paper.

Before beginning, we establish some notation. We shall be dealing with closed subsets $E$ of $\partial D$, and we shall always assume, without loss of generality, that $-1 \in E$. For such an $E, \partial D \sim E$ is the union of a collection $\left\{\left(e^{i a_{n}}, e^{i b_{n}}\right)\right\}$ of disjoint open arcs such that $-\pi \leqslant a_{n}<b_{n} \leqslant \pi$. We put $\varepsilon_{n}=b_{n}-a_{n}$ and, when $E$ has been specified, shall use the $a_{n}$ 's, $b_{n}$ 's, and $\varepsilon_{n}$ 's without further comment. Now suppose that $f$ is a continuous function defined on $D$. We put $\|f\|_{\infty}$ equal to the (possibly infinite) number $\sup \{|f(z)|: z \in D\}$ and, for $0<r<1$, write

$$
M(r, f)=\left(\frac{1}{2 \pi}\right) \int_{-\pi}^{\pi}\left|f\left(r e^{i t}\right)\right| d t .
$$

THEOREM 1. Suppose that $g$ is analytic in $D$, continuous on $\bar{D}$, and that

Received by the editors March 4, 1977.

AMS (MOS) subject classifications (1970). Primary 30A78; Secondary 46J15.

'Partially supported by NSF Grant MCS76-02267. 
$\operatorname{Re} g>0$ in $D$. Let $N$ be the number of zeroes that $g$ has on $\partial D$. Then $N-1 \leqslant 2 \pi\left\|g^{\prime}\right\|_{\infty}^{2} /|g(0)|^{2}$.

Proof. Evidently we may assume that $\left\|g^{\prime}\right\|_{\infty}<+\infty$ so that, in particular, $g^{\prime} \in H^{1}$ and $g$ is absolutely continuous on $\partial D$ with $d g\left(e^{i t}\right) / d t=$ $i e^{i t} \lim _{r \rightarrow 1} g^{\prime}\left(r e^{i t}\right)$ for almost every $t \in[-\pi, \pi]$. (See Theorem 3.11 in [2].) Thus

$$
\left|g\left(e^{i t}\right)-g\left(e^{i s}\right)\right| \leqslant\left\|g^{\prime}\right\|_{\infty}|t-s|, \quad-\pi \leqslant t, s<\pi .
$$

Now let $E$ be the zero set of $g$ in $\partial D$. Given $n$, it follows from (1) and $g\left(e^{i a_{n}}\right)=g\left(e^{i b_{n}}\right)=0$ that

(2) if $a_{n}<t<b_{n}$, then

$$
\left|g\left(e^{i t}\right)\right| \leqslant\left\|g^{\prime}\right\|_{\infty} \min \left\{t-a_{n}, b_{n}-t\right\} \leqslant\left\|g^{\prime}\right\|_{\infty} \varepsilon_{n} / 2 .
$$

Let $u$ and $v$ be, respectively, the real and imaginary parts of $g$. Then the real part of $1 / g$ is $u /|g|^{2}$. Thus

(3)

$$
\begin{aligned}
& \frac{u(0)}{|g(0)|^{2}}=\left(\frac{1}{2 \pi}\right) \int_{-\pi}^{\pi} \frac{u\left(e^{i t}\right)}{\left|g\left(e^{i t}\right)\right|^{2}} d t=\left(\frac{1}{2 \pi}\right) \sum_{n} \int_{a_{n}}^{b_{n}} \frac{u\left(e^{i t}\right)}{\left|g\left(e^{i t}\right)\right|^{2}} d t \\
& \geqslant\left(\frac{1}{\pi\left\|g^{\prime}\right\|_{\infty}^{2}}\right) \sum_{n}\left(\frac{1}{\varepsilon_{n}}\right)\left[\int_{a_{n}}^{\left(a_{n}+b_{n}\right) / 2} \frac{u\left(e^{i t}\right)}{t-a_{n}} d t+\int_{\left(a_{n}+b_{n}\right) / 2}^{b_{n}} \frac{u\left(e^{i t}\right)}{b_{n}-t} d t\right],
\end{aligned}
$$

where the last inequality follows from (2).

It follows from (1) that the integral

$$
\left(\frac{1}{2 \pi}\right) \int_{-\pi}^{\pi} u\left(e^{i t}\right) \cot \left(\frac{a-t}{2}\right) d t
$$

converges absolutely whenever $u\left(e^{i a}\right)=0$. In fact, the formula for conjugate functions on the circle allows us to write, in this case,

$$
v\left(e^{i a}\right)=\left(\frac{1}{2 \pi}\right) \int_{-\pi}^{\pi} u\left(e^{i t}\right) \cot ((a-t) / 2) d t+v(0)
$$

Since, in particular,

$$
u\left(e^{i a_{n}}\right)=u\left(e^{i b_{n}}\right)=v\left(e^{i a_{n}}\right)=v\left(e^{i b_{n}}\right)=0
$$

we have

$$
\int_{-\pi}^{\pi} u\left(e^{i t}\right)\left[\cot \left(\frac{a_{n}-t}{2}\right)-\cot \left(\frac{b_{n}-t}{2}\right)\right] d t=0 .
$$

Rewriting this we get

$$
\begin{array}{r}
\int_{-\pi}^{a_{n}}+\int_{b_{n}}^{\pi} u\left(e^{i t}\right)\left[\cot \left(\frac{a_{n}-t}{2}\right)-\cot \left(\frac{b_{n}-t}{2}\right)\right] d t \\
=\int_{a_{n}}^{b_{n}} u\left(e^{i t}\right)\left[\cot \left(\frac{t-a_{n}}{2}\right)+\cot \left(\frac{b_{n}-t}{2}\right)\right] d t
\end{array}
$$

To examine the right-hand side of (4), we first note that for $a_{n} \leqslant t \leqslant\left(a_{n}+\right.$ 
$\left.b_{n}\right) / 2$ we have

$$
\begin{aligned}
\left|\cot \left(\frac{b_{n}-t}{2}\right)\right| & <\cot \left(\frac{t-a_{n}}{2}\right) \\
& <\frac{\pi}{\left(t-a_{n}\right)} .
\end{aligned}
$$

Thus

$$
\begin{gathered}
\int_{a_{n}}^{\left(a_{n}+b_{n}\right) / 2} u\left(e^{i t}\right)\left[\cot \left(\frac{t-a_{n}}{2}\right)+\cot \left(\frac{b_{n}-t}{2}\right)\right] d t \\
<2 \pi \int_{a_{n}}^{\left(a_{n}+b_{n}\right) / 2 \frac{u\left(e^{i t}\right)}{t-a_{n}} d t}
\end{gathered}
$$

Similarly,

(6)

$$
\int_{\left(a_{n}+b_{n}\right) / 2}^{b_{n}} u\left(e^{i t}\right)\left[\cot \left(\frac{t-a_{n}}{2}\right)+\cot \left(\frac{b_{n}-t}{2}\right)\right] d t
$$

$$
<2 \pi \int_{\left(a_{n}+b_{n}\right) / 2}^{b_{n}} \frac{u\left(e^{i t}\right)}{b_{n}-t} d t .
$$

Now (3), (5), and (6) yield

$$
\frac{u(0)}{|g(0)|^{2}}>\frac{1}{2 \pi^{2}\left\|g^{\prime}\right\|_{\infty}^{2}} \sum_{n} \frac{1}{\varepsilon_{n}} \int_{a_{n}}^{b_{n}} u\left(e^{i t}\right)\left[\cot \left(\frac{t-a_{n}}{2}\right)+\cot \left(\frac{b_{n}-t}{2}\right)\right] d t .
$$

Taking into account (4), we have

$$
\begin{aligned}
& u(0) /|g(0)|^{2} \\
& \geqslant \frac{1}{2 \pi^{2}\left\|g^{\prime}\right\|_{\infty}^{2}} \sum_{n} \frac{1}{\varepsilon_{n}} \int_{-\pi}^{a_{n}}+\int_{b_{n}}^{\pi} u\left(e^{i t}\right)\left[\cot \left(\frac{a_{n}-t}{2}\right)-\cot \left(\frac{b_{n}-t}{2}\right)\right] d t \\
& =\frac{1}{2 \pi^{2}\left\|g^{\prime}\right\|_{\infty}^{2}} \int_{-\pi}^{\pi} u\left(e^{i t}\right)\left\{\sum_{n} \frac{1}{\varepsilon_{n}}\left[\cot \left(\frac{a_{n}-t}{2}\right)-\cot \left(\frac{b_{n}-t}{2}\right)\right]\right. \\
& \left.\cdot \chi_{\left[-\pi, a_{n}\right] \cup\left[b_{n}, \pi\right]}(t)\right\} d t,
\end{aligned}
$$

where $\chi_{\left[-\pi, a_{n}\right] \cup\left[b_{n}, \pi\right]}$ is the characteristic function of the set $\left[-\pi, a_{n}\right] \cup\left[b_{n}, \pi\right]$. Now if $-\pi \leqslant t \leqslant a_{n}$ or $b_{n} \leqslant t \leqslant \pi$, it is easy to check that

$$
\cot \left(\frac{a_{n}-t}{2}\right)-\cot \left(\frac{b_{n}-t}{2}\right) \geqslant \frac{\varepsilon_{n}}{2} \text {. }
$$

Thus if $t \in\left(a_{m}, b_{m}\right)$, the quantity $\{\cdots\}$ in the last term of (7) is not less than $\sum_{n \neq m} 1 / 2$. Letting $N$ be the cardinality (a priori possibly $+\infty$ ) of the collection $\left\{\left(e^{i a_{n}}, e^{i b_{n}}\right)\right\}$, and noting that almost every $t \in[-\pi, \pi]$ is in some $\left(a_{m}, b_{m}\right)$, we see from (7) that 


$$
\frac{u(0)}{|g(0)|^{2}} \geqslant \frac{N-1}{2 \pi\left\|g^{\prime}\right\|_{\infty}^{2}}\left(\frac{1}{2 \pi}\right) \int_{-\pi}^{\pi} u\left(e^{i t}\right) d t=\frac{(N-1) u(0)}{2 \pi\left\|g^{\prime}\right\|_{\infty}^{2}} .
$$

This finishes the proof of the theorem.

COROLlaRY. If $E$ is a peak set for Lip 1, then $E$ is finite.

Proof. Suppose that $f \in \operatorname{Lip} 1$ peaks on $E$. It follows from a result of Hardy and Littlewood (see Theorem 5.1 in [2]) that $\left\|f^{\prime}\right\|_{\infty}<\infty$, so Theorem 1 applies to $g=1-f$.

Theorem 1 is a quantitative version of the following statement: if $g$ is analytic in $D$, continuous on $\bar{D}$, and has positive real part on $D$, and if $\left\|g^{\prime}\right\|_{\infty}$ is finite, then the zero set of $g$ is finite. If the hypotheses on $g$ are strengthened to require that $g^{\prime}$ be continuous on $\bar{D}$, this statement is proved in [1]. It is perhaps surprising how much more difficult the proof becomes when $f^{\prime}$ is not required to be continuous on $\bar{D}$.

The proof of our next theorem is similar to the proof of Theorem 5 in [1].

THEOREM 2. Suppose $0<\alpha<1$ and that $E \subseteq \partial D$ is a closed set of measure zero satisfying $\sum_{n} \varepsilon_{n}^{(1-\alpha) /(3-\alpha)}<+\infty$. Then $E$ is a peak set for $\operatorname{Lip} \alpha$.

Proof. Put $\gamma=2 /(3-\alpha)$ and define $\phi$ on $\partial D$ by

$$
\phi\left(e^{i t}\right)=\left\{\begin{array}{l}
\left(t-a_{n}\right)^{-\gamma}+\left(b_{n}-t\right)^{-\gamma} \text { if } a_{n}<t<b_{n}, \\
+\infty \text { if } e^{i t} \in E .
\end{array}\right.
$$

Our hypothesis $\sum_{n} \varepsilon_{n}^{(1-\alpha) /(3-\alpha)}<+\infty$ implies that the function $t \mapsto \phi\left(e^{i t}\right)$ is integrable on $[-\pi, \pi]$. Next define an analytic function $g$ on $D$ by

$$
g(z)=\frac{1}{2 \pi} \int_{-\pi}^{\pi} \phi\left(e^{i t}\right) \frac{e^{i t}+z}{e^{i t}-z} d t .
$$

The properties of $g$ that we need are these:

(i) $g$ has a continuous extension to $\bar{D} \sim E$,

(ii) $\operatorname{Re} g\left(r e^{i t}\right) \rightarrow \phi\left(e^{i t}\right)$ as $r \rightarrow 1$,

(iii) $\left|g^{\prime}(z)\right| \leqslant M[\operatorname{dist}(z, E)]^{-2}$ for some constant $M$,

(iv) $g^{\prime} \exp (-g)$ is bounded on $D$.

For (i) and (ii) see [5, p. 80]; for (iii) see [8, Lemma 2.3]; and for (iv) see [6, pp. [270-1271]. Now put $h=1 / g$ and $f=\exp (-h)$. Then $h$ has a continuous extension to $\bar{D}, h$ has positive real part on $\bar{D} \sim E$ (because $g$ does), and $h\left(e^{i t}\right)=0$ for $e^{i t} \in E$. Thus $f$ peaks on $E$ and all that remains is to show that $f \in \operatorname{Lip} \alpha$. This will be done by showing that $h^{\prime}$ (and thus $f^{\prime}$ ) belongs to the Hardy class $H^{p}$, where $p=1 /(1-\alpha)$. (See, for example, exercise 9 on p. 91 in [2].)

Now $h^{\prime}=-h^{2} g^{\prime} \exp (-g) / \exp (-g)$, so we see that $h^{\prime}$ is the quotient of two bounded analytic functions. Thus $h^{\prime}$ belongs to the Nevanlinna class $N$. But because $\exp (-g)$ is an outer function, $h^{\prime} \in N^{+}$. Thus $h^{\prime} \in H^{p}$ provided the boundary function $h^{\prime}\left(e^{i t}\right) \in L^{p}(\partial D)$. (See [2, Theorem 2.11].) Now 


$$
\begin{aligned}
\left|h^{\prime}\left(r e^{i t}\right)\right| & =\left|g^{\prime}\left(r e^{i t}\right)\right| \cdot\left|g\left(r e^{i t}\right)\right|^{-2} \\
& \leqslant M\left[\operatorname{dist}\left(r e^{i t}, E\right)\right]^{-2}\left[\operatorname{Re} g\left(r e^{i t}\right)\right]^{-2} .
\end{aligned}
$$

Thus it follows from (ii) and (iii) above that

$$
\underset{r \rightarrow 1}{\limsup }\left|h^{\prime}\left(r e^{i t}\right)\right|<M\left[\operatorname{dist}\left(e^{i t}, E\right)\right]^{-2}\left[\phi\left(e^{i t}\right)\right]^{-2} .
$$

There is a constant $K$ such that $\left[\operatorname{dist}\left(e^{i t}, E\right)\right]^{-\gamma}<K \phi\left(e^{i t}\right)$, and so

$$
\left[\operatorname{dist}\left(e^{i t}, E\right)\right]^{-2}<\left[K \phi\left(e^{i t}\right)\right]^{2 / \gamma} \text {. }
$$

When combined with (8), this implies that

$$
\left|h^{\prime}\left(e^{i t}\right)\right|<M K^{2 / \gamma}\left[\phi\left(e^{i t}\right)\right]^{(2 / \gamma)-2} \text { a.e. }
$$

Consequently,

$$
\left|h^{\prime}\left(e^{i t}\right)\right|^{p}<\text { constant } \cdot \phi\left(e^{i t}\right) \text { a.e. }
$$

This shows that $h^{\prime}\left(e^{i t}\right) \in L^{p}(\partial D)$ and so completes the proof of the theorem.

To prove our final theorem we require a lemma.

LEMMA. Let $g$ be analytic on $D$ and have positive real part. Then

$$
M(r, g)=O(\log [1 /(1-r)]) \quad \text { as } r \rightarrow 1 .
$$

Proof. This is a consequence of [3, Theorem 7].

THEOREM 3. Fix $\alpha$ with $0<\alpha<1$ and suppose $E \subseteq \partial D$ is a peak set for Lip $\alpha$. Then for each $\delta>1$ we have

$$
\sum_{n} \varepsilon_{n}^{1-\alpha}\left|\log \left(1 / \varepsilon_{n}\right)\right|^{-\delta}<+\infty
$$

Proof. Let $\delta>1$ be given. Let us assume, without loss of generality, that $b_{n}-a_{n}<\pi / 2$ for each $n$ so that if $r_{0}$ is the smallest of the numbers $\cos \left(b_{n}-a_{n}\right)$, then $0<r_{0}<1$. Since $E$ is a peak set for Lip $\alpha$, there exists $f \in \operatorname{Lip} \alpha$ having positive real part on $\partial D \sim E$ with $f\left(e^{i t}\right)=0$ for $e^{i t} \in E$. Let $K$ be a Lipschitz constant for $f$ on $\bar{D}$ so that $|f(z)-f(w)|<K|z-w|^{\alpha}$ $(z, w \in \bar{D})$. (The assumption that $f \in \operatorname{Lip} \alpha$ means that $f$ satisfies a Lipschitz condition on $\partial D$. But an old result of Hardy and Littlewood [4, Theorem 41] shows that $f$ is then Lipschitz on $\bar{D}$.)

By elementary calculus, $\int_{0}^{1} 1 /(1-r)[\log 1 /(1-r)]^{\delta} d r<+\infty$. When combined with the Lemma as applied to $g=1 / f$, this yields

$$
\int_{r_{0}}^{1} M(r, 1 / f) /(1-r)[\log 1 /(1-r)]^{1+\delta} d r<+\infty .
$$

Thus, 


$$
\begin{aligned}
+\infty & >\int_{r_{0}}^{1}\left[\int_{-\pi}^{\pi} \frac{1 /\left|f\left(r e^{i t}\right)\right|}{(1-r)[\log 1 /(1-r)]^{1+\delta}} d t\right] d r \\
& =\int_{r_{0}}^{1}\left[\sum_{n} \int_{a_{n}}^{b_{n}} \frac{1 /\left|f\left(r e^{i t}\right)-f\left(e^{i a_{n}}\right)\right|}{(1-r)[\log 1 /(1-r)]^{1+\delta}} d t\right] d r \\
& =\sum_{n} \int_{a_{n}}^{b_{n}}\left[\int_{r_{0}}^{1} \frac{d f\left(r e^{i t}\right)-f\left(e^{i a_{n}}\right) \mid(1-r)[\log 1 /(1-r)]^{1+\delta}}{\mid l}\right] d t \\
& \geqslant \sum_{n} \int_{a_{n}}^{b_{n}}\left[\int_{r_{0}}^{1} \frac{\left.K \mid r e^{i t}-e^{\left.i a_{n}\right|^{\alpha}(1-r)[\log 1 /(1-r)]^{1+\delta}}\right] d t}{d r}\right] d t . \\
& \geqslant \sum_{n} \int_{a_{n}}^{b_{n}}\left[\int_{\cos \left(t-a_{n}\right)}^{1} \frac{K \mid e^{i t}-e^{i a_{n} \mid \alpha}(1-r)[\log 1 /(1-r)]^{1+\delta}}{K}\right]
\end{aligned}
$$

(The last inequality follows from the facts $r_{0} \leqslant \cos \left(b_{n}-a_{n}\right) \leqslant \cos \left(t-a_{n}\right)$ if $a_{n}<t<b_{n}$, and $\left|r e^{i t}-e^{i a_{n}}\right| \leqslant\left|e^{i t}-e^{i a_{n}}\right|$ if $\left.\cos \left(t-a_{n}\right) \leqslant r \leqslant 1\right)$. Evaluating $\int_{\cos \left(t-a_{n}\right)}^{1} 1 /(1-r)[\log 1 /(1-r)]^{1+\delta} d r$, we see that the last sum above is equal to

$$
\begin{aligned}
(1 / K \delta) \sum_{n} \int_{a_{n}}^{b_{n}} & \frac{d t}{\left|e^{i t}-e^{i a_{n}}\right|^{\alpha}\left[\log 1 /\left(1-\cos \left(t-a_{n}\right)\right)\right]^{\delta}} \\
& \geqslant\left(\frac{1}{2^{\delta} K \delta}\right) \sum_{n} \int_{a_{n}}^{b_{n}} \frac{d t}{\left(t-a_{n}\right)^{\alpha}\left[\log \pi / \sqrt{2}\left(t-a_{n}\right)\right]^{\delta}},
\end{aligned}
$$

where in obtaining the inequality we have used the relations

$$
\left|e^{i t}-e^{i a_{n}}\right| \leqslant t-a_{n} \text { and } 1-\cos \left(t-a_{n}\right) \geqslant 2\left(t-a_{n}\right)^{2} / \pi^{2}
$$

It follows, after a change of variable, that

$$
+\infty>\sum_{n} \int_{0}^{\sqrt{2} \varepsilon_{n} / \pi} t^{-\alpha}[\log 1 / t]^{-\delta} d t
$$

Consider the equation

$$
\begin{aligned}
(d / d t)\left(t^{1-\alpha}[\log 1 / t]^{-\delta}\right) \\
=(1-\alpha) t^{-\alpha}(\log 1 / t)^{-\delta}+\delta t^{-\alpha}(\log 1 / t)^{-\delta-1}
\end{aligned}
$$

By integrating both sides of this equation from 0 to $\sqrt{2} \varepsilon_{n} / \pi$ and then summing over $n$, we obtain 


$$
\begin{aligned}
\left(\frac{\sqrt{2}}{\pi}\right)^{1-\alpha} \sum_{n} \varepsilon_{n}^{1-\alpha}\left(\log \frac{\pi}{\sqrt{2} \varepsilon_{n}}\right)^{-\delta} \\
=(1-\alpha) \sum_{n} \int_{0}^{\sqrt{2} \varepsilon_{n} / \pi} t^{-\alpha}\left(\log \frac{1}{t}\right)^{-\delta} d t \\
+\delta \sum_{n} \int_{0}^{\sqrt{2} \varepsilon_{n} / \pi} t^{-\alpha}\left(\log \frac{1}{t}\right)^{-\delta-1} d t .
\end{aligned}
$$

Each sum on the right-hand side of this equation is finite because of (9). Thus so is the left-hand side. It follows easily that $\Sigma_{n} \varepsilon_{n}\left|\log 1 / \varepsilon_{n}\right|^{-\delta}+\infty$. This completes the proof of the theorem.

In conclusion, we conjecture that the condition $\sum_{n} \varepsilon_{n}^{1-\alpha}<+\infty$ is necessary and sufficient for a closed subset $E$ of $\partial D$ having measure 0 to be a peak set for Lip $\alpha$.

\section{REFERENCES}

1. D. J. Caveny and W. P. Novinger, Boundary zeros of functions with derivative in $H^{p}$, Proc. Amer. Math. Soc. 25 (1970), 776-780.

2. P. L. Duren, Theory of $\boldsymbol{H}^{p}$ spaces, Academic Press, New York, 1970.

3. G. H. Hardy and J. E. Littlewood, Some properties of conjugate functions, J. Reine Angew. Math. 167 (1931), 405-423.

4. __ Some properties of fractional integrals. II, Math. Z. 34 (1932), 403-439.

5. K. Hoffman, Banach spaces of analytic functions, Prentice-Hall, Englewood Cliffs, N.J., 1962.

6. B. A. Taylor and D. L. Williams, Ideals in rings of analytic functions with smooth boundary values, Canad. J. Math. 22 (1970), 1266-1283.

7. __ The peak sets of $A^{m}$, Proc. Amer. Math. Soc. 24 (1970), 604-606.

8. Z Zeros of Lipschitz functions analytic in the unit disc, Michigan Math. J. 18 (1971), 129-139.

Department of Mathematics, Florida State University, Tallahassee, Florida 32306 